Tohoku J. Exp. Med., 2004, 203, 133-139

Case Report

\title{
Intracranial Invasive Aspergillosis Originating in the Sphenoid Sinus: A Successful Treatment with High-Dose Itraconazole in Three Cases
}

\author{
Takahiko Yamanoi, Ken Shibano, Tomoko Soeda, Akihiko Hoshi, Yutaka \\ Matsuura, Yoshiniro Sugiura, Kazuhiro Endo and Teij Yamamoto \\ Department of Neurology, Fukushima Medical University School of Medicine, \\ Fukushima 960-1295
}

Yamanoi, T., Shibano, K., Soeda, T., Hoshi, A., Matsuura, Y., Sugiura, Y., Endo, K. and Yамамото, T. Intracranial Invasive Aspergillosis Originating in the Sphenoid Sinus: A Successful Treatment with High-Dose Itraconazole in Three Cases. Tohoku J. Exp. Med., 2004, 203 (2), 133-139 — We report three cases of intracranial aspergillosis originating in the sphenoid sinus in immunocompetent patients. The patients presented with an orbital apex syndrome in that a unilateral loss of vision and cranial nerve III palsy were seen in all cases and a contralateral involvement was also seen in one case. Despite the initial treatment with a conventional dose of itraconazole (ITCZ, $200 \mathrm{mg}$ /day), the neurological deficits failed to improve and the granulomatous inflammation was not suppressed. Therefore, we treated with a combination of a high dose of ITCZ at 500-1000 mg/day (16-24 mg/ $\mathrm{kg} /$ day) and amphotericin B (AMPH-B) at $0.5 \mathrm{mg} / \mathrm{kg} / \mathrm{day}$, in conjunction with a pulse dose of methylprednisolone at $1000 \mathrm{mg} /$ day. Two cases responded favorably in that the ocular movements completely recovered, and their maximum serum concentrations of the hydroxy ITCZ were $7816 \mathrm{ng} / \mathrm{ml}$ and $5370 \mathrm{ng} / \mathrm{ml}$. However, the other case worsened, despite ITCZ treatment at $16 \mathrm{mg} / \mathrm{kg} / \mathrm{day}$, and the serum concentration of the hydroxy ITCZ was $3863 \mathrm{ng} / \mathrm{ml}$. The surgical decompression of the cavernous sinus via an extradural approach was performed, and the dose of ITCZ was increased to $24 \mathrm{mg} / \mathrm{kg} /$ day. The resulting serum concentration of the hydroxy ITCZ was 4753 $\mathrm{ng} / \mathrm{ml}$, and the outcome of this case has been favorable. These results suggest that a high blood level of the hydroxy ITCZ (more than $4500 \mathrm{ng} / \mathrm{ml}$ ) is a prerequisite for the successful treatment of intracranial aspergillosis and that the combination treatment of ITCZ with AMPH-B would be preferred. The concomitant use of steroid and/or surgical decompression should be considered, if the invasiveness is not wellcontrolled in spite of intensive medical therapy. —— intracranial aspergillosis; itraconazole; antifungal therapy; fungus infection (C) 2004 Tohoku University Medical Press

Received January 7, 2004; revision accepted for publication April 9, 2004.

Address for reprints: Takahiko Yamanoi, Department of Neurology, Fukushima Medical University School of Medicine, 1 Hikarigaoka, Fukushima 960-1295, Japan.

e-mail: yamanoi@fmu.ac.jp 
Although aspergillus is occasionally a cause of chronic sphenoid sinusitis, the intracranial extension from the sinus is a rare event. Once the intracranial invasion of infection occurs, this form of aspergillus infection is difficult to control with conventional antifungal and surgical therapies and the mortality is notoriously high (Tsuboi et al. 1988; Denning 1996; Naim et al. 1996; Okada et al. 1998; Takahashi et al. 1998; Murthy et al. 2000; Hurst et al. 2001).

Although itraconazole (ITCZ), a triazole agent that possesses a high antifungal activity against aspergillus, is available, the conventional dose that is utilized for deep-seated aspergillosis is not clinically beneficial for this particular form. Recent reports indicate that an extremely high blood concentration of ITCZ that is achieved with a large oral dose may improve the prognosis (Kloss et al. 1991; Sánchez et al. 1995; Imai et al. 1999).

We recently experienced three consecutive cases of intracranially invaded aspergillosis that originated in the sphenoid sinus in immunocompetent individuals. Here, we report that treatment with the high dose of ITCZ was successful.

\section{CASe Reports}

Case 1.

A 61-year-old man presented with impaired eye movements and a decreased vision in the right eye of 3 months duration. A mass was detected in the right orbital apex by magnetic resonance images (MRIs), and steroid therapy was attempted since the orbital apex syndrome was thought to be due to a nonspecific granulomatous inflammation. However, an aspergillus granuloma was identified by sphenoid sinus exploration, and intravenous fluconazole (200 mg/day) and oral ITCZ (200 $\mathrm{mg} /$ day) were instituted. Despite the treatment, the vision and ocular motility deteriorated over the next two weeks, and the patient was referred to our service.

Neurological examination upon admission showed an exophthalmos and a total loss of vision with optic disc atrophy in the right eye. Ocular movements were restricted in all directions indicating a complete paralysis of the III, IV, and VI cranial nerves (Fig. 1, Left), and the corneal sensation was lost in the right eye. The serum and spinal fluid concentration of $\beta$-D glucan were 35.4 and $10.0 \mathrm{pg} / \mathrm{ml}$. MRIs revealed a mass fully occupying the right sphenoid sinus, and the extension of the mass into the right orbital apex and the right cavernous sinus. The cavernous sinus portion of the right internal carotid artery was narrowed. The mass was irregularly enhanced by intravenous gadolinium (Fig. 2, Left).

The oral ITCZ was increased to $900 \mathrm{mg} /$ day

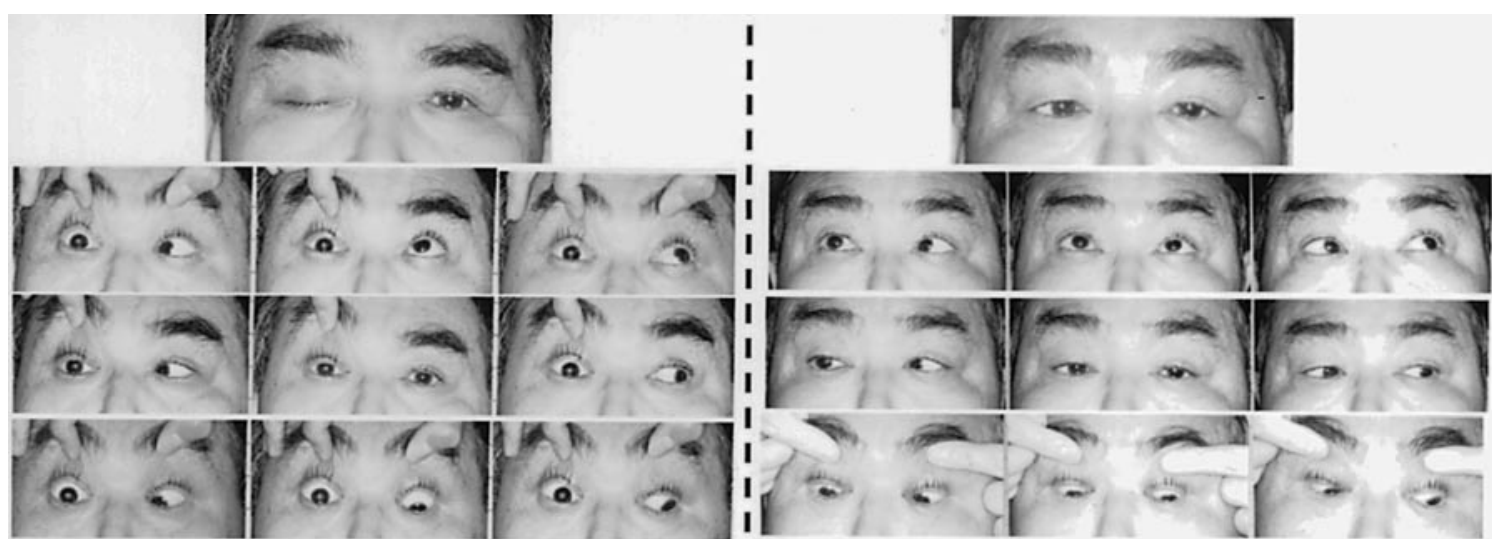

Fig. 1. Left panel: Eye movements of Case 1 in nine gaze positions prior to the high dose ITCZ. Ocular movements were restricted in all directions in the right eye.

Right panel (three months later): Marked improvement was noted in the right ocular movements. (Agreement is obtained for publishing the photograph from this patient.) 

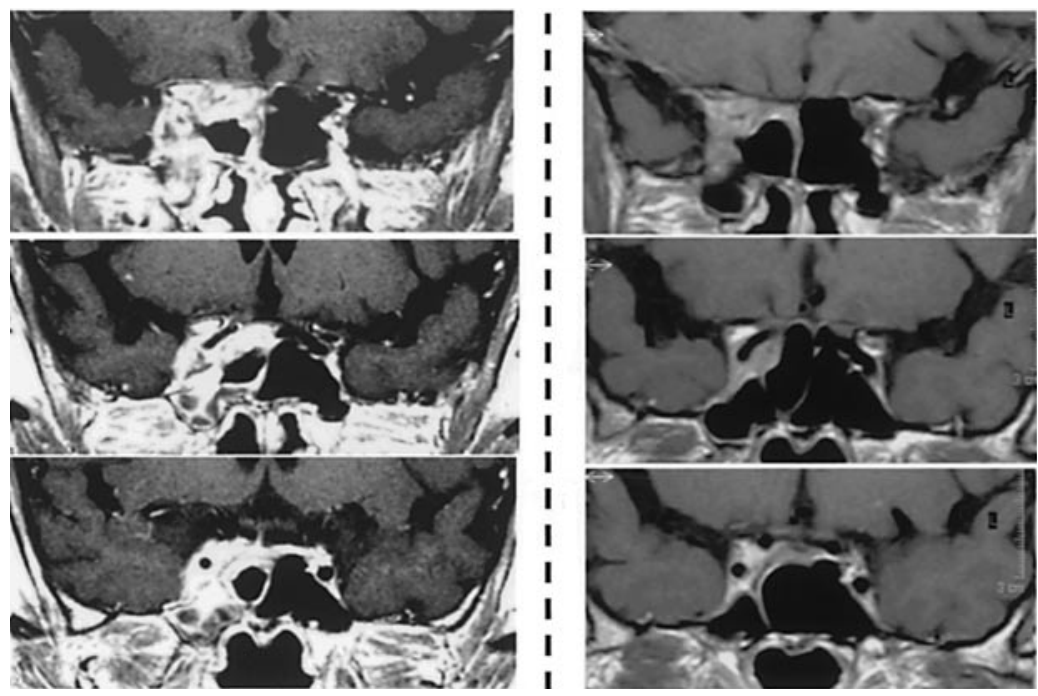

Fig. 2. Left panel: MRI of the cavernous sinus. T1-weighted images with gadolinium.

A mass in the sphenoid sinus, irregularly enhanced by gadolinium-DTPA, extended into the right cavernous sinus and the orbital apex. The right internal carotid artery was narrowed.

Right panel (eight months later): MRIs with gadolinium enhancement disclosed that the right orbital apex and cavernous sinus were only minimally enhanced, but a small mass remained discernable in the cavernous sinus.

$(16 \mathrm{mg} / \mathrm{kg} / \mathrm{day})$, and was administered in combination with intravenous amphotericin $\mathrm{B}$ (AMPH-B) at $25 \mathrm{mg} /$ day $(0.5 \mathrm{mg} / \mathrm{kg} /$ day). In addition, a pulse dose of intravenous methylprednisolone sodium succinate ( $1 \mathrm{~g}$ /day for 3 days) was added when the reducing tendency of the granuloma was confirmed. Then the rapid improvement of the ocular movements was apparent. Oral prednisolone $(30 \mathrm{mg} /$ day) was followed, and slowly tapered off over the next 6 months. AMPH-B was discontinued 3 months later when creatinine clearance fell below $30 \mathrm{ml} / \mathrm{min}$. Oral ITCZ (900 mg/day) was given for 3 months, and the serum concentration of hydroxy ITCZ rose to $7816 \mathrm{ng} / \mathrm{ml}$. The level of ITCZ was reduced to $800 \mathrm{mg} /$ day over the next 5 months. Profound hypokalemia due to AMPH-B nephrotoxicity necessitated potassium chloride supplementation during AMPH-B treatment. Three months later, right eye movements fully returned (Fig. 1, Right), although the vision remained totally lost. The serum concentration of $\beta$-D glucan became $8.8 \mathrm{pg} / \mathrm{ml}$.

Eight months after the high dose ITCZ thera- py, the patient developed a profound drug-induced hepatitis (total serum bilirubin up to $22.6 \mathrm{mg}$ / $100 \mathrm{ml})$, presumably due to an extremely high serum concentration of ITCZ. The liver function was normalized by the withdrawal of ITCZ. The patient is currently neurologically intact except for the right vision, which has never returned. MRIs with gadolinium disclosed that the right orbital apex and cavernous sinus were only minimally enhanced, and a small mass was discernible in the cavernous sinus (Fig. 2, Right). No recurrence was found three years post treatment.

\section{Case 2}

A 79-year-old woman developed impaired eye movements and a decreased vision in the right eye over the period of a month. Afterward, the ocular movements and vision in the left eye began to deteriorate. Soon, a total loss of vision occurred in both eyes. The serum and spinal fluid concentration of $\beta$-D glucan were 26.1 and 2.5 $\mathrm{pg} / \mathrm{ml}$. MRIs revealed a mass occupying the right sphenoid sinus with an extension into the right cavernous sinus and the right orbital apex. The 


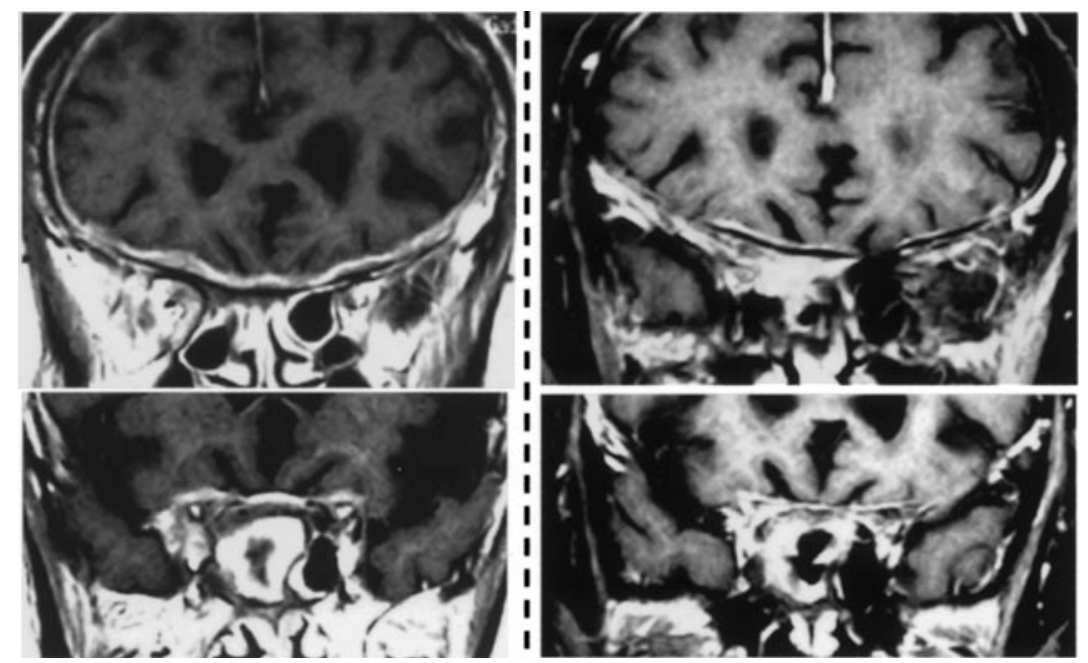

Fig. 3. Left panel: MRIs of the cavernous sinus. T1-weighted images with gadolinium. A Gd-enhanced mass occupies the right sphenoid sinus, and extends into the right cavernous sinus and the right orbital apex. The dura mater was thickened and enhanced by gadolinium. The optic nerves appear involved bilaterally.

Right panel (three months later): The mass became smaller and the thickness and enhancement of the dura mater was improved.

dura mater surrounding the sinus was thickened and was enhanced by intravenous gadolinium. Inflammation was discernible in the contralateral orbital apex as well (Fig. 3, Left). An aspergillus granuloma was identified by sphenoid sinus exploration.

Oral ITCZ at $500 \mathrm{mg} /$ day $(16 \mathrm{mg} / \mathrm{kg} /$ day $)$ was initiated, together with intravenous AMPH-B at $15 \mathrm{mg} /$ day $(0.5 \mathrm{mg} / \mathrm{kg} /$ day $)$. A pulse dose intravenous methylprednisolone sodium succinate (1 $\mathrm{g}$ /day for 3 days) was also started, followed by oral prednisolone at $30 \mathrm{mg} /$ day. This regimen was tapered down over the next 3 months. The AMPH-B administration was continued for 3 months until creatinine clearance became less than $30 \mathrm{ml} / \mathrm{min}$. When oral ITCZ at $500 \mathrm{mg} /$ day had been administered for 3 months, the serum concentration of the hydroxy ITCZ reached 5370 $\mathrm{ng} / \mathrm{ml}$. Five months later, the right eye movements were fully recovered and the left vision improved to $20 / 400$, although the right vision remained totally lost. The serum concentration of $\beta$-D glucan became $4.5 \mathrm{pg} / \mathrm{ml}$. MRIs disclosed that a small mass remained discernible in the right cavernous sinus, but the dura mater was no longer thickened or enhanced (Fig. 3, Right).

\section{Case 3}

A 57-year-old man that suffered with impaired eye movements and a decreased vision in the right eye for 3 months was thought to have orbital apex syndrome, and a sphenoid sinus mass was identified (Fig. 4, A). Aspergillus granuloma was identified when the sphenoid sinus was explored surgically. The conventional dose of ITCZ $250 \mathrm{mg} /$ day $(6.6 \mathrm{mg} / \mathrm{kg} /$ day $)$ was started, however, the aspergillus granuloma rapidly expanded to the right cavernous sinus.

Three months after initiation of antifungal therapy, he was referred to our service. Neurological examination showed an exophthalmos and a total loss of vision with optic disc atrophy in the right eye. The right eye was fixed with cranial nerves III, IV, and VI completely paralyzed. Corneal sensation was lost on the right eye. The serum and spinal fluid concentration of $\beta$-D glucan were 43.6 and $61.8 \mathrm{pg} / \mathrm{ml}$.

The right internal carotid artery was observed by intracranial magnetic resonance angiography to be occluded, although no evidence of ce- 

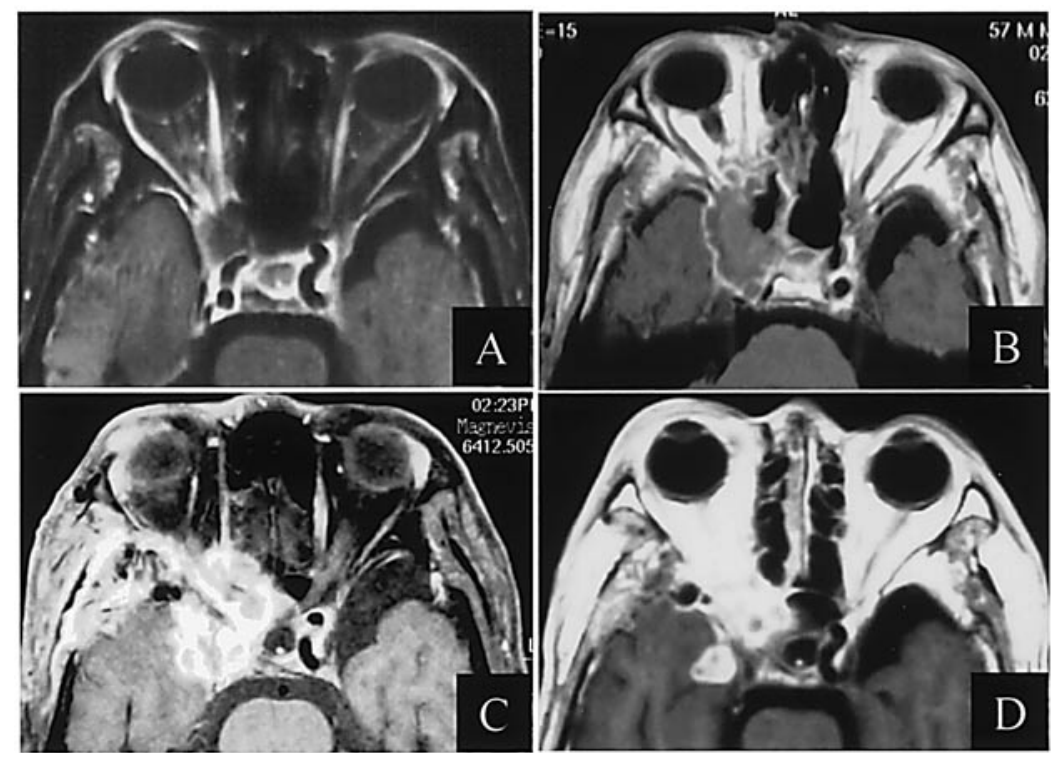

Fig. 4. A (before treatment): Axial postcontrast T1-weighted images with gadolinium enhancement displayed a mass in the right orbital apex.

B (three months after initiation of antifungal therapy): The mass rapidly expanded and occluded the right internal carotid artery.

C (six weeks after the surgical decompression): The posterior part of the mass increased and invaded into the hippocampus.

D (eight months after the surgical decompression): The mass that invaded into the hippocampus became smaller.

rebral infarction was found. The mass was enhanced irregularly by intravenous gadolinium (Fig. 4, B).

Oral ITCZ at $800 \mathrm{mg} / \mathrm{day}(19 \mathrm{mg} / \mathrm{kg} /$ day $)$, intravenous AMPH-B at $25 \mathrm{mg} /$ day $(0.5 \mathrm{mg} / \mathrm{kg} /$ day) and a pulse dose of intravenous methylprednisolone sodium succinate ( $1 \mathrm{~g} /$ day for 3 days) was started. After 3 days, the prednisolone was switched to $30 \mathrm{mg} /$ day orally. The administration of AMPH-B was continued for 2 months. In spite of the treatment, neuroimagings revealed that the expansion and invasiveness of the mass were more apparent and the intracavernous mass had advanced into the temporal fossa. Therefore, an attempt was made to decompress the mass in the cavernous sinus by the extradural approach via a right frontotemporal craniotomy. The surgery reduced the mass of the aspergilloma in the anterior cavernous portion. However, the posterior portion soon increased in size and was clearly invaded into the hippocampus after surgery (Fig. 4, C).
The dose of ITCZ was increased to $1000 \mathrm{mg} /$ day (24 mg/kg/day), with which the serum concentration of hydroxy ITCZ rose to $4753 \mathrm{ng} / \mathrm{ml}$. The patient occasionally developed an uncinate type of complex partial seizure, however, he was neurologically intact and stable, except that the right vision that had not recovered. Eight months after surgical decompression, MRIs with gadolinium enhancement disclosed that the right cavernous sinus and the invaded mass into the hippocampus remained enhanced, but were much smaller (Fig. 4, D).

\section{Discussion}

Although ITCZ shows in vitro and in vivo activity against aspergillus species, the treatment of cerebral aspergillosis has been discouraging using the conventional dose for deep mycosis (Perrin et al. 1989; Denning and Stevens 1990; Kim et al. 1993). In a review by Sánchez et al (1995), three of four patients with cerebral asper- 
gillosis that were treated with a high dose of ITCZ ( $800 \mathrm{mg} /$ day or $16 \mathrm{mg} / \mathrm{kg} /$ day) survived, while seven of eight patients treated with $400 \mathrm{mg}$ /day ITCZ died. Consistent with those results, the conventional dose of ITCZ failed to control intracranial aspergilloma in our two cases, while the high dose of ITCZ resulted in improvement. In case 1, drug-induced liver injury occurred at 8 months of treatment with the high dose ITCZ, which necessitated the discontinuance of this therapy. However, to date, the flare-up of aspergillosis has been suspended for three years without antifungal therapy, and we speculate that the disease has been cured. In case 3, ITCZ of 1000 $\mathrm{mg} /$ day $(24 \mathrm{mg} / \mathrm{kg} /$ day) controlled the aspergilloma, while $800 \mathrm{mg} /$ day (19 mg/kg/day) ITCZ did not. Previous reports have emphasized the importance of monitoring the blood concentration of ITCZ, rather than the dose. From our experience, we assume that a hydroxy ITCZ blood level of $4500 \mathrm{ng} / \mathrm{ml}$ or above would be required to improve the outcome of invasive intracranial aspergillosis, and, if the dose fails to control the growth, a higher dose must be tried and maintained for 6-12 months or longer.

Previous reports also suggest that the intracranial aspergilloma is resistant to any mode of antifungal therapy (Dupont 1990; Kreisel 1994), and, often, the condition is ultimately fatal in spite of this magnitude of high dose therapy. Thus, we attempted a combination with high dose ITCZ and AMPH-B in conjunction with methylprednisolone sodium succinate pulse therapy. The administration of steroid in these cases is controversial. The rationale behind its use is that the optic nerve, as well as the ocular motor nerves, that are clearly compromised by this granuloma, may be damaged if the inflammation surrounding the nerve is not controlled. In case 1, although the improvement of ocular movements was not clear despite of the reduction of the size of granuloma by high dose antifungal therapy, the rapid neurological improvements were observed by the methylprednisolone sodium succinate pulse therapy. Thus, the use of steroid with full dose of antifun- gal agents was an important determinant of favorable neurological outcome. In fact, the ocular motor nerves became functional in all three cases presented here, but the optic nerve function remained unimproved, except for the left eye of case 2. Anatomically the optic canal courses over the thin-walled superior lateral aspect of the sphenoid sinus, and forms the eminentia optica. Therefore, the optic nerve may be first involved in our cases. In the $3 \mathrm{rd}$ case, the surgical decompression of the cavernous sinus, which is also a controversial issue, did not appear to be beneficial in the short run, since the granuloma had invaded into the hippocampus. However, the ultimate outcome of the case appears favorable. The rich blood supply to the granuloma, which was enhanced after decompression, may have played a beneficial role in allowing a better penetration of ITCZ to the granuloma. The high blood level of ITCZ must be monitored with extreme caution, since it might invite profound drug-induced hepatitis. The role of AMPH-B, when combined with ITCZ, remains to be elucidated.

In conclusion, intracranial invasive aspergilloma is a life-threatening condition that may be treated with a high blood concentration of ITCZ. The concomitant use of steroid and/or surgical decompression should be considered when the invasiveness is not well-controlled.

Lastly, the new antifungal agents such as voriconazole and micafungin have been developed recently to treat aspergillosis. Although these new regimens appear promising for invasive aspergillosis (Herbrecht et al. 2002), further studies are required in order to provide the safety and efficacy for intracranial invasive aspergillosis.

\section{References}

Denning, D.W. \& Stevens, D.A. (1990) Antifungal and surgical treatment of invasive aspergillosis: review of 2,121 published cases. Rev. Infect. Dis., 12, 1147-1201.

Denning, D.W. (1996) Therapeutic outcome in invasive aspergillosis. Clin. Infect. Dis., 23, 608-615.

Dupont, B. (1990) Itraconazole therapy in aspergillo- 
sis: study in 49 patients. J. Am. Acad. Dermatol., 23, 607-614.

Herbrecht, R., Denning, D.W., Patterson, T.F., Bennett, J.E., Greene, R.E., Oestmann, J.W., Kern, W.V., Marr, K.A., Ribaud, P., Lortholary, O., Sylvester, R., Rubin, R.H., Wingard, J.R., Stark, P., Durand, C., Caillot, D., Thiel, E., Chandrasekar, P.H., Hodges, M.R., Schlamm, H.T., Troke, P.F. $\&$ de Pauw, B. (2002) Voriconazole versus amphotericin B for primary therapy of invasive aspergillosis. N. Engl. J. Med., 347, 408-415.

Hurst, R.W., Judkins, A., Bolger, W., Chu, A. \& Loevner, L.A. (2001) Mycotic aneurysm and cerebral infarction resulting from fungal sinusitis: imaging and pathologic correlation. AJNR. Am. J. Neuroradiol., 22, 858-863.

Imai, T., Yamamoto, T., Tanaka, S., Kashiwagi, M., Chiba, S., Matsumoto, H. \& Uede, T. (1999) Successful treatment of cerebral aspergillosis with a high oral dose of itraconazole after excisional surgery. Intern. Med., 38, 829-832.

Kim, D.G., Hong, S.C., Kim, H.J., Chi, J.G., Han, M.H., Choi, K.S. \& Han, D.H. (1993) Cerebral aspergillosis in immunologically competent patients. Surg. Neurol., 40, 326-331.

Kloss, S., Schuster, A., Schroten, H., Lamprecht, J. \& Wahn, V. (1991) Control of proven pulmonary and suspected CNS aspergillus infection with itraconazole in a patient with chronic granulomatous disease. Eur. J. Pediatr., 150, 483-485.

Kreisel, W. (1994) Therapy of invasive aspergillosis with itraconazole: our own experiences and review of the literature. Mycoses., 37, Suppl., 2, 42-51.
Murthy, J.M., Sundaram, C., Prasad, V.S., Purohit, A.K., Rammurti, S. \& Laxmi, V. (2000) Aspergillosis of central nervous system: a study of 21 patients seen in a university hospital in South India. J. Assoc. Physicians. India., 48, 677-681.

Naim Ur Rahman, Jamjoom, A., al Hedaithy, S.S., Jamjoom, Z.A., al Sohaibani, M.O. \& Aziz, S.A. (1996) Cranial and intracranial aspergillosis of sino-nasal origin. Report of nine cases. Acta Neurochir. (Wien), 138, 944-950.

Okada, Y., Shima, T., Nishida, M., Yamane, K. \& Yoshida, A. (1998) Subarachnoid hemorrhage caused by Aspergillus aneurysm as a complication of transcranial biopsy of an orbital apex lesion - case report. Neurol. Med. Chir. (Tokyo), 38, 432-437.

Perrin, C., Boutard, P., Reman, O., Lecacheux, C. \& Chapon, F. (1989) Fatal cerebral aspergillosis in acute megakaryoblastic leukemia. Arch. Fr. Pediatr., 46, 45-48.

Sánchez, C., Mauri, E., Dalmau, D., Quintana, S., Aparicio, A. \& Garau, J. (1995) Treatment of cerebral Aspergillosis with itraconazole: do high doses improve the prognosis? Clin. Infect. Dis., 21, 1485-1487.

Takahashi, Y., Sugita, Y., Maruiwa, H., Hirohata, M., Tokutomi, T. \& Shigemori, M. (1998) Fatal hemorrhage from rupture of the intracranial internal carotid artery caused by aspergillus arteritis. Neurosurg. Rev., 21, 198-201.

Tsuboi, K., Higuchi, O., Nose, T. \& Maki, Y. (1988) Intracranial Aspergillus granuloma originating in the sphenoidal sinus-case report. Neurol. Med. Chir. (Tokyo), 28, 1014-1019. 